\title{
Femtosecond laser-controlled self-assembly of amorphous-crystalline nanogratings in silicon
}

Daniel Puerto, ${ }^{1, *}$ Mario Garcia-Lechuga, ${ }^{1}$ Javier Hernandez-Rueda, ${ }^{1}$ Adianez Garcia-Leis, ${ }^{2}$ Santiago SanchezCortes, ${ }^{2}$ Javier Solis, ${ }^{1}$ and Jan Siegel ${ }^{1, *}$

1. Laser Processing Group, Instituto de Optica, CSIC, Serrano 121, 28006 Madrid, Spain

2. Instituto de Estructura de la Materia, CSIC, Serrano 121, 28006 Madrid, Spain

*corresponding authors: puerto@io.cfmac.csic.es, j.siegel@csic.es

\begin{abstract}
Self-assembly (SA) of molecular units to form regular, periodic extended structures is a powerful bottomup technique for nanopatterning, inspired by nature. SA can be triggered in all classes of solid materials, for instance, by femtosecond laser pulses leading to the formation of laser-induced periodic surface structures (LIPSS) with a period slightly shorter than the laser wavelength. This approach, though, typically involves considerable material ablation, which leads to an unwanted increase of the surface roughness. We present a new strategy to fabricate high-precision nanograting structures in silicon, consisting of alternating amorphous and crystalline lines, with almost no material removal. The strategy can be applied to static irradiation experiments and can be extended into one and two dimensions by scanning the laser beam over the sample surface. We demonstrate that lines and areas with parallel nanofringe patterns can be written by an adequate choice of spot size, repetition rate and scan velocity, keeping a constant effective pulse number $\left(N_{\text {eff }}\right)$ per area for a given laser wavelength. A deviation from this pulse number leads either to inhomogeneous or ablative structures. Furthermore, we demonstrate that this approach can be used with different laser systems having widely different wavelengths $(1030 \mathrm{~nm}$, $800 \mathrm{~nm}, 400 \mathrm{~nm})$, pulse durations (370 fs, $100 \mathrm{fs})$ and repetition rates $(500 \mathrm{kHz}, 100 \mathrm{~Hz}$, single pulse) and that the grating period can also be tuned by changing the angle of laser beam incidence. The grating structures can be erased by irradiation with a single nanosecond laser pulse, triggering recrystallization of the amorphous stripes. Given the enormous differences in electrical conductivity between the two phases our structures could find new applications in nanoelectronics.
\end{abstract}

\section{Introduction}

Laser-Induced Periodic Surface Structures (LIPSS), were discovered decades ago [1] and are formed via self-assembly in a broad range of materials, including metals, semiconductors and dielectrics. There is an ongoing debate about the exact formation mechanism of LIPSS but the most established view is that the incident laser light interferes with a surface wave [2]. This wave can be either light scattered at a rough surface or surface plasmon polaritons (SPP), and their interference with the directly incident light causes a modulated intensity distribution, being imprinted into the material. LIPSS have mainly been observed in the ablation regime, leading to a strong periodic modulation of the surface topography $[3,4,5]$ and only few works report on other mechanisms for LIPSS formation $[6,7,8]$.

Silicon is as a material of enormous technological interest and its dominance in the electronics industry is undisputed. This undiminished technological interest has also caused that the response of Si to laser irradiation has been studied thoroughly over decades [9,10,11,12,13], turning it arguably into one of the world's best-characterized material. There are numerous fundamental studies (experimental and theoretical) of LIPSS formation in Si under fs laser irradiation [14,15,16], investigating the formation mechanism and the influence of laser parameters on the ripple period and orientation. Moreover, there are technologically-oriented studies aiming at the fabrication of large area gratings by exploiting this effect [17]. One of silicon's most appealing characteristics for applications is the existence of two structurally different solid phases (crystalline and amorphous), having very different physical properties. The amorphous phase can be obtained by laser melting accompanied by fast quenching, whereas recrystallization can be obtained by either thermal or laser annealing. The use of ultrashort pulses (picoand femtosecond) allows triggering non-thermal phase transitions [18,19] which can lead to different 
solidification pathways. Besides the pulse duration, other parameters have been identified to strongly influence the phase change, namely the laser wavelength and number of pulses required, and how both parameters influence the thickness of the amorphous layer formed [20]. Yet, to the best of our knowledge, so far there is no study on the possibility to attempt the fabrication of crystalline-amorphous LIPSS via self-assembly, as opposed to grating structures mainly based on ablation/reorganization.

A first indication of this possibility has been reported recently by Katsumata et al., who have observed regions of self-assembled amorphous-crystalline nanogratings in GeTe thin films after irradiation with multiple fs pulses [7]. This material belongs to the class of so-called phase change materials [21,22], exploited mainly for optical and electrical data storage. Yet, the authors achieved only micron-sized $(5 \mu \mathrm{m} \times 5 \mu \mathrm{m})$ gratings and no strategies were proposed for upscaling these to larger structures for possible applications. In this paper we report on the formation of self-assembled phase change gratings in silicon upon multiple pulse irradiation. By carefully choosing the spatial pulse overlap, this phenomenon can be extended into two dimensions using beam scanning, leading to the formation of extended amorphous-crystalline nanograting structures. We demonstrate that this strategy can be adapted for different laser parameters.

\section{Experimental setup}

The main laser system used for the experiments was a fiber-based fs laser-amplifier (Satsuma, Amplitude Systems), which delivers laser pulses with a pulse duration of $\Delta \mathrm{t} \approx 370 \mathrm{fs}$ at a central wavelength of $1030 \mathrm{~nm}$ and a repetition rate of $500 \mathrm{kHz}$. The output Gaussian beam has a nominal diameter of $5.5 \times 6.2 \mathrm{~mm}\left(1 / \mathrm{e}^{2}\right)$. The maximum pulse energy is $10 \mu \mathrm{J}$ and can be adjusted by means of a lambda-half $\left(\lambda_{2}\right)$ wave-plate combined with a double thin film polarizer. A second $\lambda_{2}$ wave-plate is used to control the polarization state of the pulse incident on the sample, which is a critical parameter in defining the ripple orientation. The laser beam passed a galvo mirror scanning system (SCANcube, Scanlab) combined with an F-theta lens $(f=100 \mathrm{~mm})$ for scanning the focused beam over the silicon surface. The focused spot beam diameter $d$ at the sample plane was characterized using the method described by Liu [23], yielding $d_{\text {small }}=15.8 \mu \mathrm{m}\left(1 / \mathrm{e}^{2}\right)$ without pinhole and $d_{\text {large }}=33.6 \mu \mathrm{m}\left(1 / \mathrm{e}^{2}\right)$ with a pinhole of $3.5 \mathrm{~mm}$ placed $50 \mathrm{~cm}$ in front of the scan head. During the experiments, the scan speed of the beam at the surface was changed from $v_{\text {scan }}=0.5 \mathrm{~m} / \mathrm{s}$ up to $3 \mathrm{~m} / \mathrm{s}$. For static irradiations, the beam position was kept constant, and the pulse number selected by a gating signal applied to the laser driver. The samples were commercial $\langle 100\rangle$ oriented crystalline silicon wafers with p-doping (boron, resistivity 1-5 Ohm-cm).

The second laser-irradiation system was a Ti:Sa laser amplifier system (Spitfire Pro, Spectra Physics) operating at a repetition rate of $100 \mathrm{~Hz}$ and a central wavelength of $800 \mathrm{~nm}$, which could be frequency-doubled to $400 \mathrm{~nm}$ using a BBO-crystal (Barium Borate). The Silicon sample was mounted on a three-axis motorized stage and irradiated by a focused laser beam, incident on the sample at an angle $\theta$ $=53^{\circ}$. The sample was inspected in real-time with an in-situ microscope [24]. Ex-situ analysis of optical and topographic changes in the processed surface were performed with an optical microscope (OM, Zeiss Axiophot, 100x objective lens, N.A. 0.9, $460 \mathrm{~nm}$ or $800 \mathrm{~nm}$ LED-illumination) and an atomic force microscope (AFM, Agilent 5100), respectively.

\section{Result and discussion}

Figure 1 shows the optical microscopy (100x objective lens, N.A. 0.9, $460 \mathrm{~nm}$ illumination) images of the silicon surface after static irradiation with different number of pulses, a spot size of $d_{\text {large }}=$ $33.6 \mu \mathrm{m}$ and fluence of $F=0.70 \mathrm{~J} / \mathrm{cm}^{2}$. As can be seen, the first pulse already induces a weak reflectivity increase within a circular area, with respect to the non-irradiated crystalline region. A reflectivity increase at visible illumination wavelength is indicative of the formation of a thin amorphous layer [12]. After the second pulse, the reflectivity increase is much enhanced, indicating a thickness increase of the amorphous layer. Most noteworthy is the presence of reflectivity nano-fringes, similar to those observed in GeTe by Katsumata et al. [7], who showed them to be the result of an amorphous-crystalline nanopattern. An important difference is the number of pulses required to observe these fringes, 300 for GeTe compared to only two in our case. Furthermore, the fringes in Si almost disappear after irradiation with a third pulse, 
which indicates that the window for optimum fringe contrast, determined by local fluence and pulse number, is very narrow.
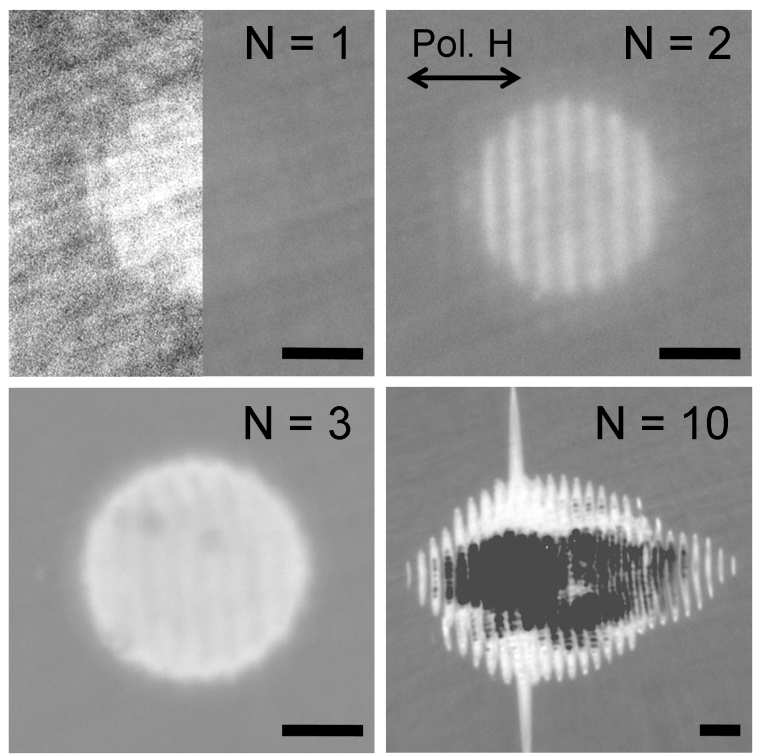

Figure 1: Optical micrographs of the silicon surface after static irradiation with different pulse number $N$ of a laser with $\lambda=$ $1030 \mathrm{~nm}$ and $\tau=370 \mathrm{fs}$. The image contrast is the same in all cases, except the left part of the image at $N=1$, where it has been adjusted to illustrate the weak reflectivity increase. The arrow indicates the orientation of the laser polarization. The scale bar corresponds to a length of $3 \mu \mathrm{m}$. Notice that the field of view of the image at $N=10$ is larger than for the other images.

Fig. 1 also shows the sample after exposure to 10 pulses, featuring ablative ripples. Interestingly, the diffraction effect of these ripples on incoming pulses can be appreciated by the presence of amorphous-crystalline fringes outside the circular amorphous region, along the horizontal axis. Such a radially non-circular ripple extension has been observed by Han et al. [25] who were able to attribute it to be a consequence of SPP scattering using FTDT modeling. The presence of a grating based on ablative ripples in the spot center facilitates coupling of laser light to a SPP wave, which otherwise would be not allowed because of the mismatch of their wave vectors. As a consequence, the electric field distribution gets elliptical with the long axis aligned along the polarization direction. While SPP theoretically can only be coupled and propagate at a dielectric/metal interface, it has also been shown to occur in semiconductors and metals through laser-induced free electron generation, turning them transiently into a metal-like state [14].

In a next step we have investigated the feasibility of writing lines of alternating amorphous and crystalline nanofringes by moving the laser beam over the sample surface. Two different approaches were tested: Extending the (few) fringes by scanning along the fringe orientation (Fig. 2a), or adding new (short) fringes by scanning along the polarization direction (Fig. 2b). It is evident that only the second strategy, scanning along the polarization direction, is successful. The underlying reason is related to the results found in Fig. $1(\mathrm{~N}=10)$, which showed effects of SPP scattering along the polarization direction, extending beyond the laser-modified region. We have illustrated this effect schematically in Fig 2 . It emerges that the second strategy is beneficial for the formation of very homogeneous and periodic grating structures, since it incubates a fringe structure along the scan direction before the actual laser spot passes and triggers the phase transition. This result suggests that not only a grating made of ablative ripples facilitates coupling of a SPP wave but also an amorphous-crystalline grating, possibly acting as a phase grating.

An indication of the phase change nature of the LIPSS formed is the reflectivity increase of the bright stripes formed. The real and imaginary part of the refractive index of the amorphous phase of Si is systematically higher than for the crystalline phase throughout the visible range [26], which implies an increase in reflectivity upon formation of a thin amorphous surface layer. We have investigated this aspect by performing optical microscopy at different illumination wavelengths $(460 \mathrm{~nm}$ Fig $2 \mathrm{~b}$ and 800 nm Fig. 2c), confirming this behavior. In order to unambiguously verify the amorphous structure of the 
bright stripes we have performed confocal Micro-Raman measurements, using laser excitation at $442 \mathrm{~nm}$ ( $1 \mathrm{~mW}$ power) and focusing down to a spot diameter of $\approx 1 \mu \mathrm{m}$. While this spot size is too large to allow for a measurement of a single amorphous stripe (500 $\mathrm{nm}$ width) it does allow recording an average spectrum of both crystalline and amorphous regions. The red curve in Fig. 2(d) shows the presence of a broad shoulder with its peak centered at $473 \mathrm{~cm}^{-1}$, characteristic for amorphous silicon [12]. The Raman band can be better resolved by measuring at a laterally more extended amorphous region, such as one of the bright regions in Fig. 2(a), leading to the blue curve in Fig. 2(d). The existence of the amorphous band becomes even clearer in comparison to a spectrum recorded at a region not exposed to the scanning laser beam, leading to the characteristic peak centered at $520.5 \mathrm{~cm}^{-1}$ of crystalline $\mathrm{Si}$, with a pronounced dip in the region of the amorphous band. The fact that the spectrum in the fringe region still shows a strong contribution from crystalline Si has two reasons. First, the already mentioned larger excitation spot size compared to the width of an amorphous stripe. Secondly, the thickness of the amorphous surface layer is only about $60 \mathrm{~nm}$ [27], compared to focal depth of about $2 \mu \mathrm{m}$ of the confocal Raman microscope, effectively collecting much of the Raman signal produced in the crystalline regions underneath the thin amorphous layer.

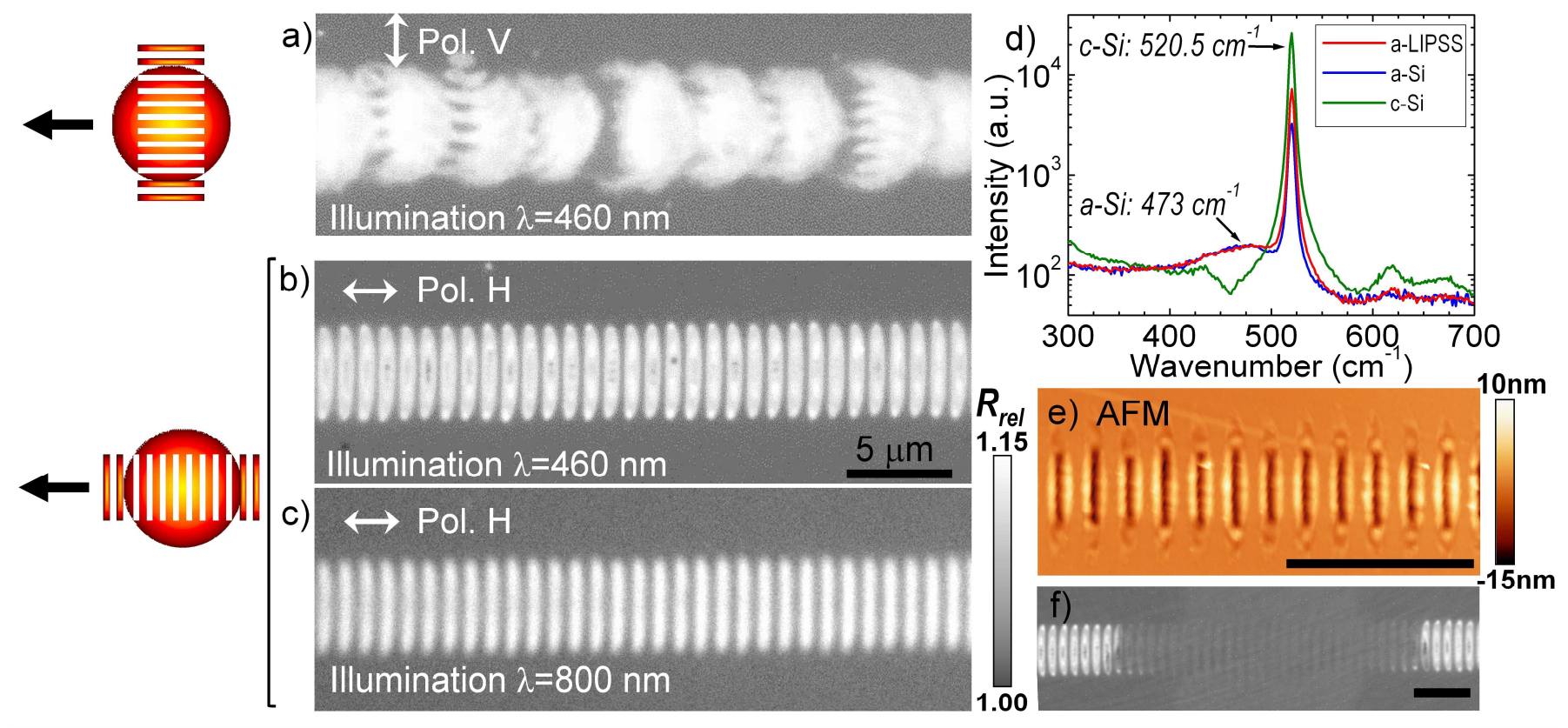

Figure 2: (a-c) Optical micrographs of amorphous-crystalline nanofringes written by moving the laser beam $(\lambda=1030 \mathrm{~nm}, \tau$ $=370 \mathrm{fs}, 500 \mathrm{kHz}$ pulses, $\left.F=0.61 \mathrm{~J} / \mathrm{cm}^{2}\right)$ at $v_{\text {scan }}=1.1 \mathrm{~m} / \mathrm{s}$ over the Si surface, using vertical (a) and horizontal $(b-c)$ polarization. The images $(b)$ and $(c)$ correspond to the same region but have been recorded with different illumination wavelength: (b) $460 \mathrm{~nm}$ and (c) $800 \mathrm{~nm}$. The scale grey bar indicate the relative reflectivity of the images (b, $460 \mathrm{~nm})$ and (c, $800 \mathrm{~nm}$ ). The sketches to the left illustrate the effect of surface plasmon polariton (SPP) scattering and its influence on the scan direction (black arrow). The Gaussian laser intensity distribution is represented by a colour-graded disk. White stripes correspond to amorphous stripes formed whereas coloured stripes correspond to local field distribution maxima produced by interference of SPP with incident laser light. (d) Raman spectra recorded in the center of the line of fringes in (b) ( $a$-LIPSS), in a bright region of (a) (a-Si) and in an unexposed region of the sample (c-Si). (e) AFM map of the amorphous-crystalline nanofringe structure of the (b) image. (f) Optical micrograph of a nanofringe line partially erased by exposure to a single nanosecond laser pulse. The scale bar in (e-f) corresponds to $5 \mu \mathrm{m}$.

Figure 2e shows a topography map of these nanofringe structures of the $2 b$ image measured by AFM. Two main observations can be made. First, it is confirmed that the amorphous stripes are not interconnected and evidence is provided that the region between amorphous stripes is unchanged, i.e. crystalline. Second, each amorphous stripe consists of an outer elevated region and an inner depression, with a total maximum modulation of $20 \mathrm{~nm}$. Such low value clearly points towards the absence of a strong ablation process but suggests the presence of evaporation. It is important to distinguish between strong ablation and evaporation, especially when studying ultrafast laser-matter interaction processes. As opposed to evaporation, which involves only minimal mass removal, ablation with fs laser pulses 
normally involves plasma formation and countless other complex processes, including ejection of droplets, nanoparticles, ions, which lead to considerable mass removal. Our result is also consistent with the mostly theoretical work reported by Tsibidis et al. on static LIPSS formation in sub-ablation conditions [28]. The authors take into account surface evaporation, defined by the lattice reaching temperature of 0.9 times the critical temperature. As a result, their model predicts a surface depression of less than $20 \mathrm{~nm}$, surrounded by a small elevation, in excellent agreement with our observations.

An interesting feature of the amorphous-crystalline grating structure is the possibility to erase them via re-crystallisation of the amorphous stripes. It is known that amorphous silicon can be crystallized by irradiation with long pulses [29] inducing melting accompanied by a relatively slow solidification process compared to irradiation with ultrashort laser pulses. To demonstrate this ability we have performed a separate experiment in which we exposed a small region of a grating line structure to a single nanosecond laser pulse $(800 \mathrm{~nm}, 8 \mathrm{~ns})$ of appropriate fluence. Fig. 2 (f) shows that the bright stripes (amorphous) are almost completely vanish within the irradiated region, demonstrating erasure via recrystallization.

Important parameters in the phase change fringes formation process are the pulse number and overlap. We have studied these for two different spot sizes by changing the scan velocity (Fig. 3). The laser parameters were kept fixed and peak fluences of $F=0.61 \mathrm{~J} / \mathrm{cm}^{2}$ (small spot) and $F=0.64 \mathrm{~J} / \mathrm{cm}^{2}$ (large spot) were used. In the former case, the small spot, we observe that strong ablation (appreciable as darkening) is induced for velocities lower than $0.8 \mathrm{~m} / \mathrm{s}$ (high pulse overlap), accompanied by an increase of the line width. In contrast, for velocities higher than $1.4 \mathrm{~m} / \mathrm{s}$ the overlap between consecutives pulses is too small to support formation of phase change fringes. Only within a small range of velocities (1-1.2 $\mathrm{m} / \mathrm{s}$ ) the formation of homogeneous amorphous nanofringes over long distances is observed. For a large spot size, the behavior is essentially the same but with velocities scaled by approximately a factor of two, which is necessary to achieve the same overlap, since the spot size ratio is $d_{\text {large }} / d_{\text {small }}=2.1$. Here, the optimum range of velocities to achieve homogeneous fringe formation is around $2-2.5 \mathrm{~m} / \mathrm{s}$.
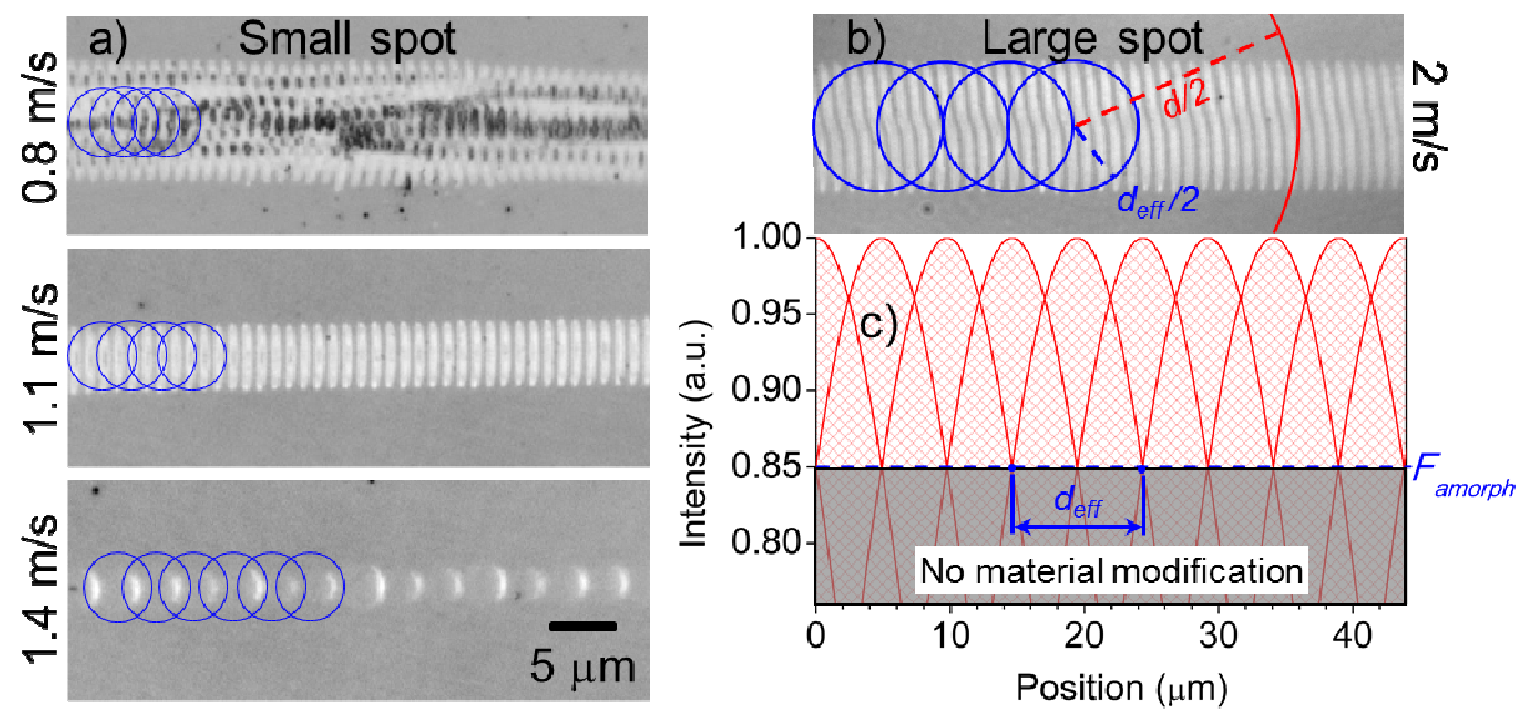

Figure 3: Optical micrographs of lines written at different speeds (see labels) with horizontally polarized laser pulses written under conditions as in Fig 2. Laser spot size is $d_{\text {small }}=15.8 \mu \mathrm{m}(\mathrm{a})$ and $d_{\text {large }}=33.6 \mu \mathrm{m}(\mathrm{b})$. The blue circles mark the position of individual effective spot diameter $\left(d_{e f f}\right)$ in each case, whose separations are determined by the scan speed. The red circle section corresponds to $d_{\text {large }} / 2(c)$ Sketch of the spatial positions of the incident Gaussian pulses (large spot) for a pulse overlap $N_{\text {eff }}=2$, indicating the intensity threshold for amorphization (see text for details).

In order to quantify the optimum pulse overlap we use the transversal extension of best fringe structure formed as criteria for defining an "effective spot diameter" $\left(d_{\text {eff }}\right)$ for each case. The values obtained are $d_{\text {eff-small }}=4.9 \mu \mathrm{m}$ and $d_{\text {eff-large }}=9.7 \mu \mathrm{m}$. These diameters are much smaller than the corresponding Gaussian spot diameters $(d)$ and represent a fluence threshold $\left(F_{\text {amorph }}\right)$ of around $0.85 \mathrm{x}$ $F_{\text {peak fluence }}$ (Fig. 3b). Figure 3a-b includes their lateral extension and relative positions in each case, with their separation being determined by the scan speed. It can be seen that for increasing speeds the pulse 
overlap is reduced. The sketch in Fig. $3 \mathrm{c}$ illustrates the intensity conditions for pulse overlap in scanning conditions, highlighting the small intensity window for homogeneous amorphous LIPSS formation.

\begin{tabular}{|c|c|c|c|c|c|}
\hline $\begin{array}{c}\text { Laser } \\
\text { parameters }\end{array}$ & $\begin{array}{c}\text { Spot size } d \\
(\mu \mathrm{m})\end{array}$ & $\begin{array}{c}\text { Peak fluence } \\
\left(\mathrm{mJ} / \mathrm{cm}^{2}\right)\end{array}$ & $\begin{array}{c}\text { Effective spot } \\
\text { size deff }(\mu \mathrm{m})\end{array}$ & $\begin{array}{c}\text { Optimum scan speed } \\
v_{\text {scan }}(\mathrm{m} / \mathrm{s})\end{array}$ & $\begin{array}{c}\text { Pulse number } N_{\text {eff }} \\
\text { for fringe formation }\end{array}$ \\
\hline $\begin{array}{c}15.8 \\
(\text { moving) }\end{array}$ & 0.61 & 4.9 & 1.1 & 2.2 \\
\cline { 2 - 6 } & $\begin{array}{c}33.6 \\
(\text { moving) }\end{array}$ & 0.64 & 9.7 & 2 & 2.4 \\
\cline { 2 - 6 } & $\begin{array}{c}33.6 \\
\text { (static) }\end{array}$ & 0.73 & 13 & 0 & 2 \\
\hline $800 \mathrm{~nm}, 100 \mathrm{~Hz}$ & $\begin{array}{c}100 \times 167 \\
(\text { moving) }\end{array}$ & 0.15 & $13.5 \times 22.5$ & $5^{*} 10^{-4}$ & 4.5 \\
\hline $400 \mathrm{~nm}, 100 \mathrm{~Hz}$ & $\begin{array}{c}59.2 \times 98.4 \\
\text { (moving) }\end{array}$ & 0.04 & $11.7 \times 19.5$ & $1.5^{*} 10^{-4}$ & 13 \\
\hline
\end{tabular}

Table 1: Conditions leading to the formation of homogeneous amorphous-/crystalline fringes upon fs laser irradiation.

In order to extend our irradiation strategy to two dimensions, we have performed laser scanning of parallel lines. Figure 4 show two examples for different line spacings. For a spacing of $9.9 \mu \mathrm{m}$ (Fig. 4 , left) an excellent reproducibility of the fringes can be appreciated, each line showing the same appearance. In order to achieve interconnection of lines and extend fringes the line spacing should in principle be equal to the line width $(4.9 \mu \mathrm{m})$. Yet, the positioning resolution of the scan head we use is discrete, with steps of $0.9 \mu \mathrm{m}$. In order to avoid line overlap we therefore chose a spacing of $5.4 \mu \mathrm{m}$. As can be seen in Fig. 4 (right), this spacing leads to connection of fringes although there are minor signs of line overlap, leading in some positions to darkening (onset of ablation) at the joints. Using a scan head with higher positioning accuracy, this problem could possibly be avoided. The bending of fringes apparent in Fig. 4 (right) is a consequence of the phase shift of the laser pulse position between lines. The fact that the fringes of different lines do connect even so, despite being displaced, indicates that there is indeed a natural tendency (self-assembly) of fringes to extend along their long axis, even when writing is performed perpendicular to them. This effect has been reported in fused silica [30] but is opposite to the one found in chrome [31].
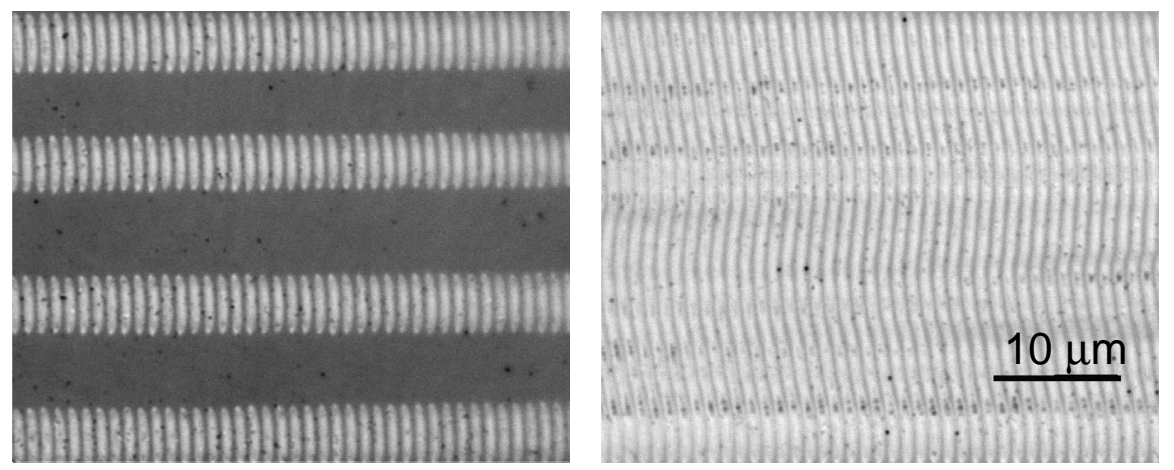

Figure 4: Optical micrographs of parallel lines with different spacings written with horizontally polarized laser pulses (1030 $\mathrm{nm}, 370 \mathrm{fs}, \mathrm{F}=0.61 \mathrm{~J} / \mathrm{cm}^{2}, 500 \mathrm{kHz}$, spot size $d=15.8 \mu \mathrm{m}, v_{\text {scan }}=1.1 \mathrm{~m} / \mathrm{s}$ ). Left: $9.9 \mu \mathrm{m}$ spacing Right: $5.4 \mu \mathrm{m} \mathrm{spacing}$.

We have calculated the effective pulse number as $N_{\text {eff }}=d_{\text {eff }} / \Delta x$, with the pulse separation $\Delta x=v_{\text {scan }} / 500$ $\mathrm{kHz}$, and the results are shown in Table 1. It can be seen that in $N_{\text {eff }}$ is comparable for both spot sizes, yielding values of around two. These values are also in good agreement with the static irradiation experiment, also included in Table 1. This agreement confirms the validity of our irradiation strategy, adapting irradiation conditions in dynamic experiments to achieve an effective pulse number of $N_{\text {eff }}=2$, being optimum for amorphous-crystalline nanofringe contrast.

Having identified the optimum irradiation strategy $\left(N_{\text {eff }} \sim 2\right)$ for a specific laser system $(1030 \mathrm{~nm}$, $370 \mathrm{fs}, 500 \mathrm{kHz}$ ), we have attempted to apply this concept to a different laser system (Ti:Sa laseramplifier, $400 \mathrm{~nm}$ or $800 \mathrm{~nm}, 100 \mathrm{fs} 100 \mathrm{~Hz}$ ) and different irradiation conditions (laser incident at an angle $\theta=53^{\circ}$ ), yielding elliptical spot sizes of $d_{400 \mathrm{~nm}}=59.2 \times 98.4 \mu \mathrm{m}$ and $d_{800 \mathrm{~nm}}=100 \times 167 \mu \mathrm{m}$. Figure 5 a shows an OM image of fringes created at $400 \mathrm{~nm}, F=0.04 \mathrm{~J} / \mathrm{cm}^{2}$ and a scan velocity of $v_{\text {scan }}=150$ $\mu \mathrm{m} / \mathrm{s}$. The fringe period is $1.8 \mu \mathrm{m}$, i.e. much larger than the laser wavelength $(400 \mathrm{~nm})$, which is due to the 
large irradiation angle [3]. Following the same reasoning as before to determinate the optimum pulse overlap, we extract an effective spot diameter of $d_{\text {eff }}=11.7 \mu \mathrm{m}$ x $22.2 \mu \mathrm{m}$ from the line width and deduce by means of the calculated spot separation a value of $N_{\text {eff }} \sim 13.5$ (c.f. Table 1). This relatively high value might seem surprising as $\mathrm{Si}\langle 100\rangle$ is known to be able to amorphize upon single pulse irradiation at appropriate fluence at this wavelength due to the short optical penetration depth [20]. Yet, it demonstrates that the self-assembly process for fringe formation requires several pulses as suggested by theory [16]. It is also worth mentioning that an increase of laser fluence led to the disappearance of fringes.

Figure $5 \mathrm{~b}$ shows an $\mathrm{OM}$ image of fringes generated at a laser wavelength of $800 \mathrm{~nm}, F=0.15$ $\mathrm{J} / \mathrm{cm}^{2}$ and a scan velocity of $v_{\text {scan }}=500 \mu \mathrm{m} / \mathrm{s}$. The fringe period is $3.7 \mu \mathrm{m}$, i.e. about two times larger than for $400 \mathrm{~nm}$. The measured effective spot diameter is $d_{\text {eff }}=13.5 \mu \mathrm{m}$ x $22.5 \mu \mathrm{m}$ and yields an optimum effective pulse number of $N_{\text {eff }} \sim 4.5$ (c.f. Table 1). The higher value compared to irradiation with $1030 \mathrm{~nm}$ is consistent with the higher number of pulses required to induce amorphization of $\mathrm{Si}\langle 100\rangle$ at this wavelength [20]. The experimentally measured fringe periods for all three wavelengths agree with the calculation of the period as a function of laser wavelength and incident angle (Fig. 5d) using the expression $p_{f w}=\lambda_{p a t} /(1-\sin \theta)$ for the interference of the scattered far field with the incident plane wave along the surface-projected forward direction [32]. The map also illustrates the possibility to fabricate structures with periods down to $350 \mathrm{~nm}$ with a laser of $400 \mathrm{~nm}$ and normal incidence. This prediction has been confirmed recently by Kumar et al. fabricating ablative LIPSS in $\mathrm{TiO}_{2}$ [5] and should in principle be possible also for the phase-change LIPSS reported here.
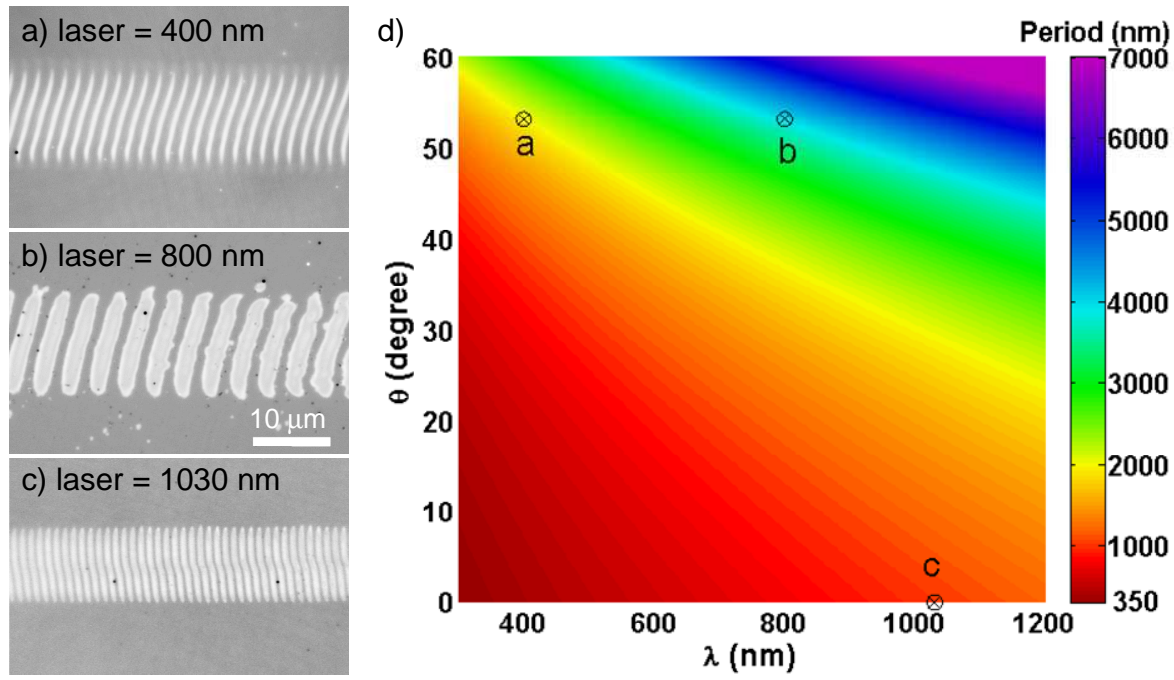

Figure 5. Optical micrographs of lines written under different laser (100 fs, $100 \mathrm{~Hz}, 400 \mathrm{~nm}(\mathrm{a})$ or $800 \mathrm{~nm}(\mathrm{~b})$, and $370 \mathrm{fs}, 500$ $\mathrm{kHz}$ and $1030 \mathrm{~nm}(\mathrm{c}))$ and irradiation conditions (incident from the right at an angle of 53 ${ }^{\circ}$, scan velocity of $v_{\text {scan }}=150 \mu \mathrm{m} / \mathrm{s}$ (a) or $v_{\text {scan }}=500 \mu \mathrm{m} / \mathrm{s}\left(\mathrm{b}\right.$,) and normal incident, $\left.v_{\text {scan }}=1.1 \mathrm{~m} / \mathrm{s}(\mathrm{c})\right)$. The beam was horizontally polarized. $(d)$ Calculation of the fringe period as function of laser wavelength and incident angle.

\section{Conclusion}

In conclusion, we have demonstrated the viability of creating phase change nanogratings via selfassembly in silicon by fs laser irradiation. This new kind of laser-induced periodic structures does not involve significant material removal and can be generated both in static irradiation and dynamic processing conditions, leading to homogeneous lines and areas of amorphous/crystalline fringes. The scan direction of the laser spot with respect to the laser polarization was found to be a crucial parameter for fringe quality, which can be explained by surface plasmon polariton scattering incubating the fringe structure along the scan direction. The laser fluence window to trigger self-assembly is relatively narrow due to the nature of the process to stay between melting and ablation threshold. The critical parameter to trigger nanograting formation was found to be the effective pulse number incident on a certain area. By adapting the effective pulse number these nanogratings can be generated with a broad range of laser wavelength and angle of incidence. Since the grating period depends strongly on these parameters, the period can be tuned over a broad range. These structures could find applications nanoelectronics [33] as $\mathrm{Si}$ is a key material in this field and the enormous difference in electrical conductivity between the 
crystalline and the amorphous phase distinguishes our nanopatterning approach. Although the present study is limited to crystalline silicon, this technique can be extended to other material that can be phaseswitched by laser pulses.

\section{Acknowledgements:}

This work has been supported by the LiNaBioFluid project of the H2020 programme of the European Commission as well as by the Spanish TEC2014-52642-C2-1-R. M.G.-L. and J.H.-R. acknowledges the grants, respectively, awarded by the Spanish Ministry of Education and the Spanish Ministry of Economy and Competiveness.

\section{References}

[1] Birnbaum M 1965 Semiconductor Surface Damage Produced by Ruby Lasers J. Appl. Phys. 36 3688-9

[2] Sipe J E, Young J F, Preston J S and van Driel H M 1983 Laser-induced periodic surface structure. I Theory Phys. Rev. B 27 1141-54

[3] Young J F, Preston J S, van Driel H M and Sipe J E 1983 Laser-induced periodic surface structure. II. Experiments on Ge, Si, Al, and brass Phys. Rev. B 27 1155-72

[4] Bonse J, Baudach S, Krüger J, Kautek W and Lenzner M 2002 Femtosecond laser ablation of silicon-modification thresholds and morphology Appl. Phys. A: Mater. Sci. Process. 74 19-25

[5] Kumar Das S, Dasari K, Rosenfeld A and Grunwald R 2010 Extended-area nanostructuring of TiO ${ }_{2}$ with femtosecond laser pulses at $400 \mathrm{~nm}$ using a line focus Nanotechnology 21155302

[6] Öktem B, Pavlov I, Ilday S, Kalaycioglu H, Rybak A, Yavas S, Erdogan M and Ilday F Ö 2013 Nonlinear laser lithography for indefinitely large-area nanostructuring with femtosecond pulses Nat. Photonics 7 897-901

[7] Katsumata Y, Morita T, Morimoto Y, Shintani T and Saiki T 2014 Self-organization of a periodic structure between amorphous and crystalline phases in a GeTe thin film induced by femtosecond laser pulse amorphization Appl. Phys. Lett. 105 031907

[8] Loeschner K, Seifert G and Heilmann A 2010 Self-organized, gratinglike nanostructures in polymer films with embedded metal nanoparticles induced by femtosecond laser irradiation J. Appl. Phys. 108073114

[9] Liu P L, Yen R, Bloembergen N and Hodgson R T 1979 Picosecond laser-induced melting and resolidification morphology on Si Appl. Phys. Lett. 34 864-6

[10] Boyd I W and Wilson J I B 1983 Laser processing of silicon Nature 303 481-6

[11] von der Linde D and Sokolowski-Tinten K 2000 The physical mechanisms of short-pulse laser ablation Appl. Surf. Sci. 154-155 $1-10$

[12] Bonse J, Brzezinka K-W and Meixner A J 2004 Modifying single-crystalline silicon by femtosecond laser pulses: an analysis by micro Raman spectroscopy, scanning laser microscopy and atomic force microscopy Appl. Surf. Sci. 221 215-30

[13] Solis J and Afonso C N 1991 Early stages of melting in Si under nanosecond laser pulse irradiation: A time-resolved study J. Appl. Phys. 69 2105-11

[14] Huang M, Zhao F, Cheng Y, Xu N and Xu Z 2009 Origin of laser-induced near-subwavelength ripples: interference between surface plasmons and incident laser ASC Nano. 3 (12) $4062-70$

[15] Bonse J, Rosenfeld A and Krüger J 2009 On the role of surface plasmon polaritons in the formation of laser-induced periodic surface structures upon irradiation of silicon by femtosecond-laser pulses J. Appl. Phys. 106104910

[16] Tsibidis G D, Fotakis C and Stratakis E 2015 From ripples to spikes: A hydrodynamical mechanism to interpret femtosecond laser-induced self-assembled structures Phys. Rev. B 92041405 (R)

[17] Le Harzic R, Dörr D, Sauer D, Neumeier M, Epple M, Zimmermann H and Stracke F 2011 Large-area, uniform, highspatial-frequency ripples generated on silicon using a nanojoule-femtosecond laser at high repetition rate Opt. Lett. 36 229-31

[18] Sokolowski-Tinten K, Bialkowski J and von der Linde D 1995 Ultrafast laser-induced order-disorder transitions in semiconductors Phys. Rev. B $\mathbf{5 1} 14186-98$

[19] Shank C V, Yen R and Hirlimann C 1983 Time-Resolved Reflectivity Measurements of Femtosecond-Optical-PulseInduced Phase Transitions in Silicon Phys. Rev. Lett. 50 454-7

[20] Izawa Y, Izawa Y, Setsuhara Y, Hashida M, Fujita M, Sasaki R, Nagai H and Yoshida M 2007 Ultrathin amorphous Si layer formation by femtosecond laser pulse Irradiation Appl. Phys. Lett. 90044107

[21] Yamada N, Ohno E, Nishiuchi K, Akahira N and Takao M 1991 Rapid phase transitions of GeTe-Sb2Te3 pseudobinary amorphous thin films for an optical disk memory J. Appl. Phys. 69 2849-56

[22] Siegel J, Gawelda W, Puerto D, Dorronsoro C, Solis J, Afonso C N, de Sande J C G, Bez R, Pirovano A and Wiemer C 2008 Amorphization dynamics of $\mathrm{Ge}_{2} \mathrm{Sb}_{2} \mathrm{Te}_{5}$ films upon nano- and femtosecond laser pulse irradiation J. Appl. Phys. 103 023516

[23] Liu J M 1982 Simple technique for measurements of pulsed Gaussian-beam spot sizes Opt. Lett. 7 196-8

[24] Hernandez-Rueda J, Siegel J, Puerto D, Galvan-Sosa M, Gawelda W and Solis J 2013 Ad-hoc design of temporally shaped fs laser pulses based on plasma dynamics for deep ablation in fused silica Appl. Phys. A Mater. Sci. Process. 112 185-9 [25] Han W, Jiang L, Li X, Liu P, Xu L and Lu Y 2013 Continuous modulations of femtosecond laser-induced periodic surface structures and scanned line-widths on silicon by polarization changes Opt. Express 21 15505-13

[26] Sadao Adachi, Optical Constants of Crystalline and Amorphous Semiconductors, Springer US (1999). 
[27] Izawa Y, Izawa Y, Setsuhara Y, Hashida M, Fujita M, Sasaki R, Nagai H and Yoshida M 2007 Ultrathin amorphous Si layer formation by femtosecond laser pulse Irradiation Appl. Phys. Lett. 90044107

[28] Tsibidis G D, Barberoglou M, Loukakos P A, Stratakis E and Fotakis C 2012 Dynamics of ripple formation on silicon surfaces by ultrashort laser pulses in subablation conditions Phys. Rev. B 86115316

[29] Thompson M O, Galvin G J, Mayer J W, Peercy P S, Poate J M, Jacobson D C, Cullis A G, and Chew N G 1984 Melting Temperature and Explosive Crystallization of Amorphous Silicon during Pulsed Laser Irradiation Phys. Rev. Lett. 52 2360

[30] Gottmann J, Wortmann D and Wagner R 2008 Manufacturing of periodical nanostructures by fs-laser direct writing Proc. SPIE 7022702202

[31] Ruiz de la Cruz A, Lahoz R, Siegel J, de la Fuente G F and Solis J 2014 High speed inscription of uniform, large-area laser-induced periodic surface structures in Cr films using a high repetition rate fs laser Opt. Express 39 2491-4

[32] Kühler P, García de Abajo F J, Solis J, Mosbacher M, Leiderer P, Afonso C N and Siegel J 2009 Imprinting the Optical Near Field of Microstructures with Nanometer Resolution Small 5 (16) 1825-9

[33] Wei Lu, and Charles M. Lieber, "Nanoelectronics from the bottom up", Nature Materials 6, 841 - 850 (2007). 